\title{
Wei-Hsin Chien \\ Severe bladder trabeculation mimics malignancy on intravenous pyelography
}

Received: 24 August 2005 / Accepted: 23 December 2005 / Published online: 15 March 2006

(C) Springer-Verlag Berlin / Heidelberg 2006

\begin{abstract}
Intravenous pyelography was performed in a woman with severe (grade IV) pelvic organ prolapse. The imaging of the bladder suggested a neoplasm, but at cystoscopy the diagnosis proved to be severe bladder trabeculation.
\end{abstract}

Keywords Bladder trabeculation - Bladder malignancy · Cystoscopy $\cdot$ Intravenous pyelography

\section{Introduction}

Bladder trabeculation has often been seen in patients with chronic lower urinary tract disease by cystoscopy, but has seldom been shown on intravenous pyelography. The author reported an interesting case of severe bladder trabeculation demonstrated on intravenous pyelography and cystoscopy.

\section{Case report}

A 40-year-old, para 7, menopausal woman complained of a protruding vaginal mass and flank pain. Severe pelvic organ prolapse grade IV was diagnosed and surgical treatment was scheduled. The pre-operative laboratory data

\footnotetext{
W.-H. Chien $(\bowtie)$

Department of Obstetrics and Gynecology,

Chang Gung Memorial Hospital,

Linkou Medical Center,

5, Fu-Shin Street,

Kwei-Shan, Taoyuan Taiwan

e-mail: a12292@.cgmh.org.tw

Tel.: +886-3-3281200

Fax: $+886-3-3288252$

Present address:

W.-H. Chien

Department of Obstetrics and Gynecology,

Taipei City Hospital,

145, Jhengjhou Rd.,

Taipei City 10341, Taiwan

e-mail: DAL04@tpech.gov.tw
}

including urinary analysis, complete blood count and blood chemistries were within normal limits. Pre-operative intravenous pyelography showed right pelviectasis and filling defect with a reticular mucosal pattern at the bladder base. A bladder neoplasm was suspected (Fig. 1a). Cystoscopy was performed for further investigation and revealed marked bladder wall trabeculation (Fig. 1b). The surgery was then performed to correct the prolapse and the patient recovered well.
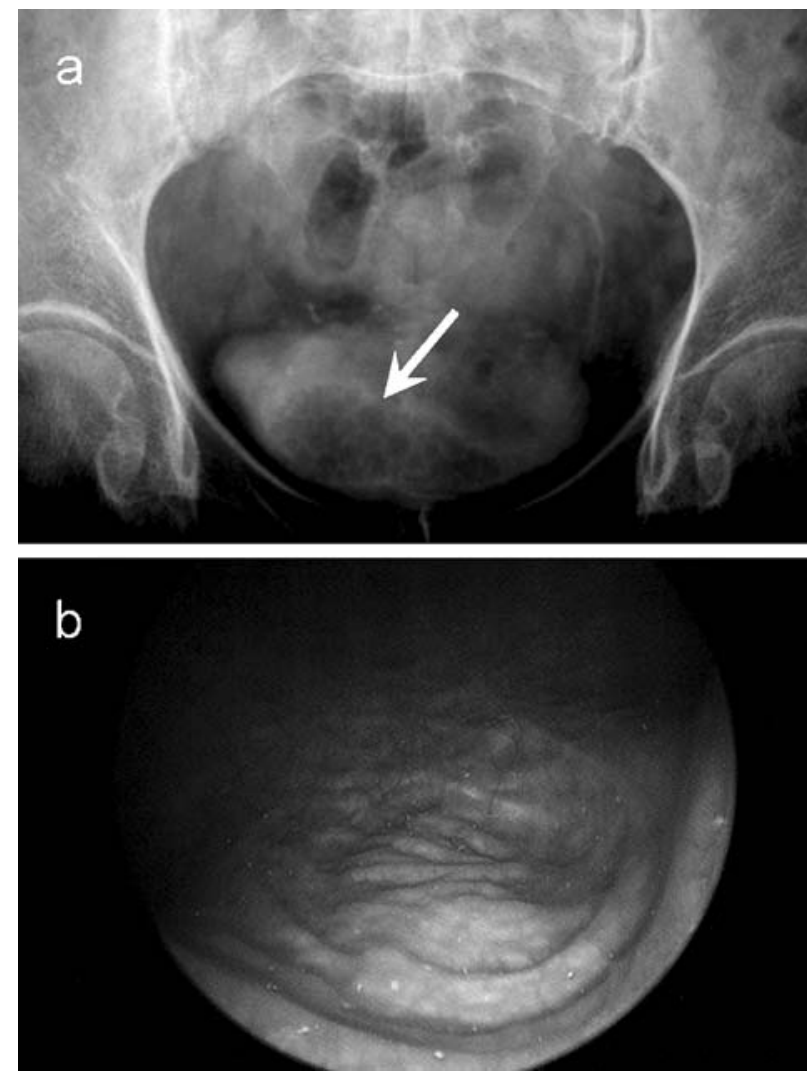

Fig. 1 a The arrow indicates filling defect with a reticular mucosal pattern on intravenous pyelography film. b The cystoscope showed significant bladder base trabeculation. 


\section{Discussion}

This patient had severe pelvic organ prolapse and the associated hydronephrosis had to be ruled out by urography [1]. In addition, she had flank pain and thus urinary stones should also be considered. Intravenous pyelography can provide information on the urinary tract and once a urinary tract filling defect has been revealed, it should be regarded as a potential malignancy [2]. In this case, the radiologic study revealed a suspected bladder neoplasm, but was unable to provide adequate bladder imaging and cystoscopy was required [3]. The accurate diagnosis was made by direct vision via cystoscopy.

In this patient, as there was no history of recurrent urinary tract infection or lower urinary tract symptoms such as urgency and frequency, the severe trabeculation might be the consequence of chronic detrusor strain resulting from an obstructed bladder outlet caused by severe pelvic organ prolapse. Therefore, in severe pelvic organ prolapse, bladder trabeculation must be included in the differential diagnosis of a bladder filling defect on intravenous pyelography.

\section{References}

1. Kobashi KC, Leach GE (2000) Pelvic prolapse. J Urol 164:1879-1890

2. Amar AD, Das S (1984) Pre-cystoscopic diagnosis of bladder tumour by modified intravenous urography. Br J Urol 56:381384

3. Amling CL (2001) Diagnosis and management of superficial bladder cancer. Curr Probl Cancer 25:219-278 